\title{
Emotional support predicts more sickness absence and poorer self assessed work ability: a two-year prospective cohort study
}

\author{
Nadine Karlsson ${ }^{1 *}$, Elisabeth Skargren ${ }^{2}$, Margareta Kristenson ${ }^{1}$
}

\begin{abstract}
Background: While back pain and stressful work environment are shown to be important causes of sickness absence the effect of psychosocial resources on sickness absence, and on self assessed work ability, is less commonly investigated. The aim of this study was to assess these associations in a two-year follow-up study.

Methods: 341 working people aged 45 to 64, randomly drawn from the population, responded to a questionnaire at baseline and at a two-year follow-up. Poisson regression was used to analyse the association of psychosocial factors (psychosocial instruments on work environment, emotional support and psychological resources) and previous back pain (low back and/or neck) at baseline with sickness absence (spells and days) at follow-up, controlling for effects of age, sex, BMI, smoking, alcohol, occupation, disease and previous sickness absence. Logistic regression was used to study the associations of psychosocial factors and previous back pain at baseline with self assessed prognosis of poor work ability six months from follow-up. Finally, a multivariate analysis tested the independent effects of previous back pain and 3 psychosocial factors derived in a factor analysis: 1. work environment; 2. emotional support; 3. psychological resources, on work ability and absence days and spells.

Results: $80 \%$ of the sickness absence spells within the last 12 months before follow-up were short-term $(\leq 14$ days). In the final model, high emotional support predicted more sickness absence spells (RR 1.36; 1.11-1.67) and days (RR 1.68, 1.22-2.31). Previous back pain (OR 2.56; 1.13-5.81), high emotional support (OR 1.58; 1.02-2.46), and low psychological resources (OR 0.62; $0.44-0.89$ ) were related to poorer self assessed prognosis of work ability at follow up.

Conclusions: In a general middle aged working population high emotional support was related to more sickness absence and also poorer self assessed prognosis of work ability. Our findings suggest that both sickness absence and self assessed work ability are dependent of life outside work and can be affected by a person's close community.
\end{abstract}

\section{Background}

Musculoskeletal disorders, primarily back pain, have been the main causes for sickness absence in Sweden [1,2], but stress-related ill health is growing rapidly as a cause of such absence [3]. Measures of stressors, in terms of psychosocial working environment, predict the occurrence of cardiovascular [4], common mental disorders [5-7], but also musculoskeletal disorders $[8,9]$, and have also

\footnotetext{
* Correspondence: nadine.karlsson@liu.se

'Department of Medical and Health Sciences, Division of Community Medicine, Social Medicine and Public Health Science, Linköping University, Linköping, Sweden

Full list of author information is available at the end of the article
}

been found to predict sickness absence in several prospective studies [10-16]. These studies, predominantly based on the demand-control-support model [17], have consistently found that low decision latitude is related to a high level of sickness absence [18] but there is no clear evidence for the effects of psychological demands or social support at work on such absence [19].

The examination of a wide-range of defined theorybased psychosocial characteristics is unusual in sickness absence research [20], and few studies have examined the association between psychosocial resources and sickness absence. An active problem-solving coping style 
has been shown to reduce the risk of sickness absence [21]. In the Whitehall II cohort, high levels of confiding/ emotional support at home was associated with a higher risk for sickness absence [22] and sickness absence, mainly the shorter, has been suggested as a coping behavior to reduce work-related stress by avoiding the workplace and thereby creating the opportunity for recuperation [23].

Whereas absence due to illness could bee seen as the negative pole in the continuum between excellent and ill health, work ability is defined as the capacity of an individual to manage gainful employment as a means of earning a living. The determinants of sickness absence and work ability are not necessarily the same [24], and few investigations have examined predictors for both sickness absence and work ability in parallel.

\section{Study aim}

The aim of this study was to investigate prospectively, over a two-year follow-up in a general middle-aged working Swedish population, the effect of psychosocial work environment, psychosocial resources and previous back pain on self-reported sickness absence and self assessed prognosis of work ability.

\section{Methods}

\section{Study design and population}

The Life Conditions, Stress, and Health (LSH) study [25-28] is a longitudinal study of socioeconomic differences in health in a population of men and women (non-patients) drawn from the county of Östergötland in the southeast of Sweden. It was based on a random sample of 1007 men and women between 45-69 years of age in 2003, as stratified by sex and age, who belonged to any of the catchment areas of ten primary health care centres in the region (response rate 62\%). Data collection at baseline (2003 to 2004) and at follow-up (2005 to 2006) included data self-reported by postal questionnaire. Exclusion criteria were serious disease and difficulties in understanding the language. Follow-up data were collected from a total of 795 men and women (response rate $79 \%$ ).

The eligible individuals for the present study were individuals who were employed both at baseline and follow-up. At baseline, 534 of the respondents in the survey were employed. 33 respondents were sick-listed at baseline and therefore excluded. Of the 501 baseline respondents, 409 returned the follow-up questionnaire (82\% response rate). Another 68 individuals were no longer employed at the time of the follow-up, resulting in a final study sample of 341 individuals (prospective cohort). At follow-up the proportion of individuals with a manual occupation was higher in non respondents than in respondents $(\mathrm{p}=0.002)$.

\section{Measurements}

\section{Self-reported sickness absence}

The self-reported number of sickness absence spells (or periods) and days were measured at baseline and at two-year follow-up by the question: "How many spells of sickness absence did you have in the last 12 months?" and "How many days of sickness absence did you have in the last 12 months?"

Measuring both the number of sickness absence spells and their duration is important, since they are only partly determined by the same variables. For example, sickness absence frequency is more influenced by an employee's task, workgroup organisation, leadership, shift work, and absence control measures, whereas sickness absence duration is more influenced by age, working conditions, sickness benefits, and access to medical care and specialists [29].

\section{Self assessed prognosis of work ability}

Information on self assessed prognosis of work ability six months from follow-up was obtained through a question posed in previous research [30]: "How much chance is there that you will be able to work within six months?" The six response categories ranged from "very unlikely" to "very likely". The answers were dichotomised, with "very likely" representing "good work ability", and the remainder categorised as "poor work ability".

\section{Psychosocial factors}

Several psychosocial factors, widely used in stress research, were measured at baseline.

Psychosocial work environment was assessed by means of three instruments: Demand-control [31], social support at work [17], and overcommitment (specific cognitive and motivational pattern of coping with demands characterized by excessive work-related commitment) [32]. Two subscales were derived from the demand-control model: psychological demands (i.e., time pressure, and conflicting demands), and decision latitude (influence over what to do and how and when to do it). Job strain was calculated as the unweighted ratio between psychological demands and decision latitude.

Psychosocial resources were assessed in two domains: social support and psychological (individual) resources.

Social support was measured in terms of emotional support, which describes the availability of affectionally close and deeper emotional relationships, and was measured by means of the psychometric instrument "Availability of Attachment" [33]. An important component of emotional support is related to self-appraisal, providing support that boosts self-esteem and encourages positive self-appraisal [34]. Emotional support differs from practical support that is manifest under many forms, including practical help and financial support. Some typical examples of items in "emotional support" question are: 
"Do you have a confident from whom you feel you really can get support?"; "Do you have a confident who can share the emotions with you when you are happy?"

Psychological (individual) resources were measured with four scales. 1. Sense of coherence [35] includes three dimensions: a) the (cognitive) ability to define life events as less stressful (comprehensibility), b) mobilising resources to deal with stressors that one encounters (manageability), and c) motivation, desire, and commitment to cope (meaningfulness). 2. Mastery [36] is often used as an equivalent to coping ability and addresses the extent to which one regards the direction of one's life as being under one's own control, in contrast to being fatalistically ruled, while 3 . self-esteem [36] refers to the positiveness of one's attitude towards oneself. The instrument 4. perceived control [37] measures how much an individual perceives that he/she can intentionally produce desired outcomes and prevent undesirable ones.

\section{Self-reported previous low back and/or neck pain}

This item was assessed at baseline through the question, "Have you had low back and/or neck pain during previous 5 years?" (yes/no)" [38].

\section{Covariates}

As covariates we included age, sex, socioeconomic status (SES), smoking (current smoker yes/no), alcohol consumption, body mass index (BMI), self-reporting a disease diagnosed by a physician (yes/no), and past sickness absence. They were assessed at baseline in the self-administered questionnaire of 2003-2004.

Socioeconomic status (SES) was based on an individual's occupation, measured according to the Swedish SEI coding system [39]. It distinguishes between manual workers (skilled or unskilled), non-manual workers (including administrators, professionals, and routine non-manual workers), and self-employed individuals (including farmers).

Alcohol consumption was categorized in three groups based on the weekly intake of alcohol in grams: low (< $80 \mathrm{~g} /$ week for women, < $110 \mathrm{~g} /$ week for men), medium (between 80-139 g/week for women, 110-169 g/week for men), and high intake ( $\geq 140 \mathrm{~g} /$ week for women, $\geq 170$ $\mathrm{g} /$ week for men).

Past sickness absence was defined as the number of sickness absence spells and days the 12 months before baseline for the analysis of sickness absence, and as sickness absence (yes/no) in the 12 months before follow-up for the analysis of work ability.

\section{Statistical analysis}

The reliability of the psychosocial scales was estimated by Cronbach's $\alpha$ internal consistency coefficient. The prospective impact of each psychosocial instrument and previous back pain at baseline was analysed with the number of sickness absence spells or days at follow-up using a Poisson regression model with a scale parameter to account for over-dispersion [40]. The analyses were adjusted successively for the following variables: 1) Model I for age and sex; 2) Model II adding lifestyle factors (BMI, smoking, and alcohol consumption), SES, and disease; 3) Model III all factors in the previous steps and adding past sickness absence. The prospective association between each psychosocial factor and previous back pain at baseline and a work ability rating of 'poor' at follow-up was analysed using a logistic regression in Models I to III described above.

A varimax-rotated principal components factor analysis [41] was performed among psychosocial factors (job strain, overcommitment, social support at work, emotional support, self-esteem, coping, sense of coherence, and perceived control) in order to derive composite dimensions of psychosocial factors, and thereby reduce the risk of reporting statistical significance due to multiple testing [42]. Finally the independent effect of three factors, derived from the factor analysis of all psychosocial instruments: work environment, emotional support and psychological resources as well as previous back pain, were analysed, simultaneously, as determinants of sickness absence (or, as the case may be poor work ability) with multivariate Poisson (or logistic) regression fully adjusted for covariates as above.

The correlation between the number of sickness absence spells and days, both at baseline and follow-up, was estimated by Spearman correlation coefficient. A significance level of $5 \%$ was considered to be statistically significant. SPSS 17 was used for factor analysis and SAS 9.1 for Poisson and logistic regressions.

The data had zero to 29 missing values for each variable, with the exception of self assessed work ability, which had 63 missing values (around $20 \%$ of participants). To retain all participants, imputed values were generated for the missing data from self assessed work ability. The 63 non responders for work ability had less sickness absence, for both spells and days, than the 278 respondents (all $p<0.01$ ). Non response on self assessed work ability was coded as good work ability and statistical analyses were conducted using the imputed dataset [43]. A complete case statistical analysis of work ability was performed, and gave similar results to that using imputed values reported in the tables.

\section{Ethical aspects}

The study was approved by the Regional Committee for Research Ethics at Linköping University (ethical file number 02-0324).

\section{Results}

\section{Characteristics of the study population}

Descriptive statistics for the prospective cohort are provided in Table 1. Mean number of sickness absence 
Table 1 Characteristics of the prospective cohort $(n=$ 341)

\begin{tabular}{|c|c|c|c|}
\hline Variable & $\begin{array}{c}\text { Total } \\
\text { (n) }\end{array}$ & $\begin{array}{l}\text { Frequency } \\
\text { (\%) }\end{array}$ & $\begin{array}{l}\text { Mean }(95 \% \\
\mathrm{Cl})\end{array}$ \\
\hline Sex & 341 & . & . \\
\hline Women & & $153(44.9 \%)$ & \\
\hline Men & & $188(55.1 \%)$ & . \\
\hline Age (in years) & 341 & . & $\begin{array}{l}52.35(51.84- \\
52.86)\end{array}$ \\
\hline Occupation (SES) & 337 & . & . \\
\hline Non manual & & $185(54.9 \%)$ & . \\
\hline Manual & & $112(33.2 \%)$ & . \\
\hline Self-employed & & $40(11.9 \%)$ & . \\
\hline Smoking, current & 332 & 69 (20.8\%) & \\
\hline BMI $\left(\mathrm{kg} / \mathrm{m}^{2}\right)$ & 340 & . & $\begin{array}{l}26.11(25.70- \\
26.52)\end{array}$ \\
\hline Alcohol consumption & 325 & . & . \\
\hline Low $^{a}$ & & $264(81.2 \%)$ & \\
\hline Medium $^{b}$ & & $36(11.1 \%)$ & . \\
\hline $\mathrm{High}^{c}$ & . & $25(7.7 \%)$ & . \\
\hline $\begin{array}{l}\text { Disease (self report of } \\
\text { diagnosis) }\end{array}$ & 341 & $171(50.2 \%)$ & \\
\hline Previous back pain & 330 & $219(66.4 \%)$ & . \\
\hline "Poor" work ability & 278 & $56(20.1 \%)$ & \\
\hline $\begin{array}{l}\text { Sickness absence spells at } \\
\text { baselinet }\end{array}$ & 341 & & $0.47(0.39-0.55)$ \\
\hline $\begin{array}{l}\text { Sickness absence spells at } \\
\text { follow-upł }\end{array}$ & 340 & . & $0.49(0.42-0.56)$ \\
\hline $\begin{array}{l}\text { Sickness absence days at } \\
\text { baselinet }\end{array}$ & 335 & & $6.59(4.83-8.35)$ \\
\hline $\begin{array}{l}\text { Sickness absence days at } \\
\text { follow-up }\end{array}$ & 340 & . & $6.96(5.09-8.83)$ \\
\hline
\end{tabular}

$\mathrm{Cl}=$ confidence interval.

${ }^{a}$ Low (< $80 \mathrm{~g} /$ week for women, $<110 \mathrm{~g} /$ week for men)

${ }^{b}$ Medium (between 80-139 g/week for women, 110-169 g/week for men)

${ }^{C} H i g h$ ( $\geq 140 \mathrm{~g} /$ week for women, $\geq 170 \mathrm{~g} /$ week for men)

† within the last 12 months before baseline

\# within the last 12 months before follow-up

spells and days at baseline and follow-up differed little over the two-year study (Table 1). The Spearman correlation coefficient between sickness absence at baseline and follow-up was 0.41 for number of spells, and 0.38 for number of days.

Most of the sick leave spells were short-term as 87/ 121 (72\%) of the individuals sick-listed within the last 12 months before baseline, and 114/142 (80\%) of the individuals sick-listed within the last 12 months before follow-up, had a total of 1-14 sick leave days.

The descriptive statistics for the different psychosocial factors are presented in Table 2.

Three factors of psychosocial scales were identified in the factor analysis: I. work environment (including job strain, overcommitment, social support at work), II. emotional support and III. psychological resources (including 1. sense of coherence, 2 . mastery, 3 . self-esteem, and 4 . perceived control). Together, the three derived components captured $64.2 \%$ of the total variance.

\section{Prospective impact of psychosocial factors and previous back pain on self-reported sickness absence}

In model I adjusted for age and sex, several factors emerged as predictors of more sickness absence spells (Table 3) and days (Table 4): occupation (non manual vs. self-employed), alcohol consumption (low vs. medium intake), disease, past sickness absence, high job strain, high emotional support, and low self-esteem. Previous back pain and low decision latitude predicted more sickness absence days but not spells.

In Model II, adjusted also for effects of demography, lifestyle factors and disease, high emotional support was the only significant predictor of more sickness absence spells and days (data not shown) and high perceived control predicted an increased number of sickness absence days. In Model III, adjusting also for previous sickness absence caused little change in most estimates.

The multivariate analysis of previous back pain and derived components of factor analysis confirmed that high levels of emotional support were related to a significant increased number of sick-leave spells $(R R=1.36$; 95\% $\mathrm{CI}=1.11-1.67 ; p=0.004)$ and days $(\mathrm{RR}=1.68$; $95 \% \mathrm{CI}=1.22-2.31 ; p=0.002)$ at follow-up (Table 5).

\section{Prospective impact of psychosocial factors and previous} back pain on self assessed prognosis of poor work ability In Model I adjusted for age and sex, several factors emerged as predictors of poor self assessed work ability (Table 6): occupation (manual vs. non manual), high BMI, past sickness absence, previous back pain, high job strain, low decision latitude, low self-esteem, low coping, low sense of coherence, and low perceived control.

In Model II, previous back pain, high job strain, low decision latitude, low self-esteem, and low coping were predictors of poor self assessed work ability (data not shown). In Model III, adjusting for past sickness absence caused little change in most estimates (see Table 6).

The multivariate logistic regression analysis of derived components of factor analysis (Table 5) showed that previous back pain (OR 2.56; 1.13-5.81; $p=0.02$ ), high emotional support (OR 1.58; 1.02-2.46; $p=0.04$ ), and low psychological resources $0.62(0.44-0.89 ; p=0.008)$ were related to poorer self assessed work ability at follow up, while the impact of work environment on work ability was no longer statistically significant (OR 1.39; 0.98$1.98 ; p=0.07$ ).

\section{Discussion}

The main finding of this study is that, in a general middle aged working population, high emotional support was related to more sickness absence and also to poorer self 
Table 2 Descriptive statistics of psychosocial scales and factor loadings for three dimensions of psychosocial scales

\begin{tabular}{|c|c|c|c|c|c|c|c|}
\hline & \multicolumn{4}{|c|}{ Descriptive statistics } & \multicolumn{3}{|c|}{ Factor loadings $^{\mathbf{b}}$} \\
\hline & $\alpha$ & n & Mean & SD & 1 & II & III \\
\hline$\%$ variance explained & & & & & 19.4 & 13.2 & 31.6 \\
\hline \multicolumn{8}{|l|}{$\begin{array}{l}\text { Psychosocial scales, } \\
\text { No. of items (score range) }\end{array}$} \\
\hline \multicolumn{8}{|l|}{ I Work environment } \\
\hline Job strain & & 324 & 0.70 & 0.17 & 0.69 & & . \\
\hline Psychological demands ${ }^{\mathrm{a}}, 5$ (5-20) & 0.69 & 327 & 13.11 & 2.51 & & . & . \\
\hline Decision latitude ${ }^{a}, 6$ (6-24) & 0.63 & 338 & 18.96 & 2.58 & . & & \\
\hline Social support at work, 6 (6-24) & 0.82 & 319 & 18.16 & 2.06 & -0.64 & & \\
\hline Overcommitment, 6 (6-24) & 0.83 & 330 & 13.17 & 4.26 & 0.76 & . & . \\
\hline \multicolumn{8}{|l|}{ II Emotional support } \\
\hline Emotional support, 6 (0-6) & 0.79 & 338 & 5.52 & 1.13 & & 0.94 & . \\
\hline \multicolumn{8}{|l|}{ III Psychological resources } \\
\hline 1. Sense of coherence, 13 (13-91) & 0.80 & 338 & 69.00 & 9.28 & . & . & 0.78 \\
\hline 2. Mastery, 7 (7-28) & 0.75 & 331 & 23.33 & 3.05 & . & . & 0.83 \\
\hline 3. Self-esteem, 10 (10-40) & 0.87 & 329 & 32.96 & 4.60 & & . & 0.84 \\
\hline 4. Perceived control, 11 (11-66) & 0.68 & 312 & 53.00 & 7.05 & . & . & 0.64 \\
\hline
\end{tabular}

Note: $\alpha=$ Cronbach's $\alpha$; SD = standard deviation.

${ }^{a}$ The derived subscales of job strain (psychological demands and decision latitude) are described statistically but were not included in the factor analysis.

${ }^{\mathrm{b}}$ Factor loadings $<0.5$ are not reported.

assessed prognosis of work ability. Another finding was that low psychological resources and previous back pain were related to a poorer self-assessed prognosis of work ability.

\section{Determinants of sickness absence}

A high level of emotional support was associated with both a higher frequency and longer duration of sickness absence. Such an association could be surprising since social support is considered protective against the development of depression in those exposed to life events [44]. Prospective studies, which control for baseline health status, consistently show increased risk of death among persons having few social relationships [45]. The association between high emotional support and increased risk of sickness absence is not surprising if such absence is seen as the effect of an "illness behaviour" rather than illness itself. High level of confiding/ emotional support may encourage empowerment, security, and perceptions of control, which legitimize taking leave from work when ill [23]. Our findings confirm and extend previous findings in sickness absence, where high levels of confiding/emotional support were associated with higher frequency of short-term and long-term sickness absence [22]. Notably the definition of long-term sickness absence is $>7$ days in the Whitehall II cohort and $>2$ weeks in our study. Both definitions are in correspondence with the social insurance system in the two countries.
Findings of an association between increased job strain and more periods of sickness absence and days when adjusted for age and sex is in line with earlier research. The component of the demand-control model associated with lower sickness absence was "high decision latitude". This confirms the result of previous research, in particular that decision latitude $[14,18]$ appeared to be a more important risk factors for sickness absence, than psychological demands and social support at work [19]. The association between job strain and sickness absence was reduced and was no longer statistically significant in the model adjusted for health behaviour and SES. The finding that previous back pain was related to the duration of sickness absence when adjusted for age and sex is consistent with previous research $[46,47]$. Poor psychosocial work conditions and physical workload are important risk factors for musculoskeletal pain [48]. The association between previous back pain and duration of sickness absence was no longer statistically significant in the model adjusted for health behaviour and SES. In both cases loss of effects after adjustment for SES can be an effect of over adjustment because of the strong relation between occupation, back pain and psychosocial factors at workplace $[10,15]$.

\section{Determinants of self assessed prognosis of work ability}

Knowledge of predictors for work ability is important for disability prevention, since work ability is an important 
Table 3 Prospective impact of psychosocial characteristics and previous back pain at baseline on sickness absence spells

\begin{tabular}{|c|c|c|c|c|c|c|}
\hline \multirow[t]{2}{*}{ Characteristic } & \multicolumn{3}{|c|}{$\begin{array}{l}\text { Age and sex adjusted } \\
\text { (Model I) }^{\dagger}\end{array}$} & \multicolumn{3}{|c|}{$\begin{array}{l}\text { Adjusted for the final model } \\
\qquad(\text { Model III) }\end{array}$} \\
\hline & $\mathbf{n}$ & RR & $95 \% \mathrm{Cl}$ & $\mathrm{n}$ & RR & $95 \% \mathrm{Cl}$ \\
\hline \multicolumn{7}{|l|}{ Occupation (SES) } \\
\hline Non-manual & 184 & 1 [ref] & & . & . & \\
\hline Manual & 112 & 1.18 & $0.89-1.57$ & & & . \\
\hline Self-employed & 40 & 0.28 & $0.12-0.62$ & & . & . \\
\hline Smoking, current & 331 & 0.79 & $0.55-1.15$ & . & . & . \\
\hline BMI & 339 & 1.01 & $0.97-1.05$ & . & . & . \\
\hline \multicolumn{7}{|l|}{ Alcohol consumption } \\
\hline Low $^{a}$ & 263 & 1 [ref] & & & & \\
\hline Medium ${ }^{b}$ & 36 & 0.41 & $0.21-0.83$ & & . & . \\
\hline $\operatorname{High}^{c}$ & 25 & 0.65 & $0.34-1.26$ & & . & . \\
\hline Disease (self report diagnosis) & 340 & 1.38 & $1.04-1.83$ & . & . & \\
\hline Past sickness absence & 340 & 1.36 & $1.21-1.51$ & . & . & . \\
\hline \multicolumn{7}{|l|}{ I Work environment } \\
\hline Job strain & 323 & 2.28 & $1.01-5.15$ & 298 & 1.50 & $0.67-3.40$ \\
\hline Psychological demands & 326 & 1.02 & $0.96-1.08$ & 299 & 1.03 & $0.97-1.09$ \\
\hline Decision latitude & 337 & 0.95 & $0.90-1.00$ & 309 & 1.00 & $0.95-1.07$ \\
\hline Social support at work & 318 & 0.95 & $0.88-1.01$ & 289 & 0.96 & $0.89-1.02$ \\
\hline Overcommitment & 329 & 1.00 & $0.97-1.03$ & 300 & 1.02 & $0.98-1.05$ \\
\hline II Emotional support & 337 & 1.42 & $1.14-1.77$ & 307 & 1.41 & $1.12-1.76$ \\
\hline \multicolumn{7}{|l|}{ III Psychological resources } \\
\hline 1. Sense of coherence & 337 & 0.99 & $0.97-1.00$ & 308 & 1.00 & $0.98-1.01$ \\
\hline 2. Mastery & 330 & 0.96 & $0.92-1.01$ & 300 & 0.99 & $0.94-1.04$ \\
\hline 3. Self-esteem & 328 & 0.96 & $0.93-0.99$ & 299 & 0.98 & $0.95-1.01$ \\
\hline 4. Perceived control & 311 & 0.99 & $0.97-1.01$ & 285 & 1.00 & $0.98-1.03$ \\
\hline Previous back pain & 329 & 0.94 & $0.69-1.28$ & 301 & 0.83 & $0.61-1.13$ \\
\hline
\end{tabular}

The rate ratio in italics denotes significance $(p<0.05)$. (Result from Model II, see text). In model III rate ratios for confounders are not reported as separate regressions were run for each of the 10 psychosocial variables and previous back pain.

$\mathrm{n}=$ number of observations used; $\mathrm{RR}=$ rate ratio; $\mathrm{Cl}=$ confidence interval.

a Low ( $<80 \mathrm{~g} /$ week for women, $<110 \mathrm{~g} /$ week for men)

${ }^{\mathrm{b}}$ Medium (between 80-139 g/week for women, 110-169 g/week for men)

'High ( $\geq 140 \mathrm{~g} /$ week for women, $\geq 170 \mathrm{~g} /$ week for men)

${ }^{\dagger}$ Adjusted for age, sex; ${ }^{\ddagger}$ age, sex, BMl, smoking, alcohol consumption, SES, disease (self report of diagnosis), and past sickness absence.

predictor of duration of sickness absence [49], and return to work [50,51].

In this study work ability was related to a broader array of determinants than sickness absence. Work ability is the self-perceived relation between work demands and individual resources, defined as health and functional ability, education and competence, values and attitudes [52,53]. The association of psychosocial factors at work (high job strain and low decision latitude) and poor self assessed prognosis of work ability in the model adjusted for age, sex, lifestyle factors, SES, disease and past sickness absence (Table 6) is consistent with previous research $[54,55]$. However in the multivariate logistic regression adjusted additionally for previous back pain, emotional support and psychological resources (Table 5), the association between psychosocial work environment and poor work ability was no longer statistically significant.
Previous back pain was related to reduced self assessed prognosis of work ability in all models proposed (Tables 5 and 6). Back disorders constitute one of the most common causes behind long-term sickness absence and disability pension in Sweden [46,47]. Persistent musculoskeletal pain has been shown to be a predictor of reduced work ability [56]. If activity aggravates the pain (such as with heavy physical work load), and the individual avoids or reduces his activities, then pain may lead to disability. Cognitive function, and overall health were related to work ability in patients with chronic musculoskeletal pain [57].

Low individual psychological resources (coping and selfesteem) were related with self assessed prognosis of poor work ability (Tables 5 and 6). Coping and self-esteem are closely related to self-efficacy [58]. Self-efficacy, which is defined as confidence in being able to carry out a set of 
Table 4 Prospective impact of psychosocial characteristics and previous back pain at baseline on sickness absence days

\begin{tabular}{|c|c|c|c|c|c|c|}
\hline \multirow[t]{2}{*}{ Characteristic } & \multicolumn{3}{|c|}{$\begin{array}{l}\text { Age and sex adjusted } \\
\text { (Model II) }^{\dagger}\end{array}$} & \multicolumn{3}{|c|}{$\begin{array}{l}\text { Adjusted for the final model } \\
\qquad \text { (Model III) }^{\ddagger}\end{array}$} \\
\hline & $\mathrm{n}$ & RR & $95 \% \mathrm{Cl}$ & $\mathrm{n}$ & RR & $95 \% \mathrm{Cl}$ \\
\hline \multicolumn{7}{|l|}{ Occupation (SES) } \\
\hline Non-manual & 184 & 1 [ref] & . & . & & . \\
\hline Manual & 112 & 1.43 & $0.98-2.07$ & & & \\
\hline Self-employed & 40 & 0.32 & $0.12-0.89$ & . & . & . \\
\hline Smoking, current & 331 & 1.14 & $0.74-1.78$ & . & . & . \\
\hline BMI & 339 & 1.03 & $0.98-1.07$ & & & \\
\hline \multicolumn{7}{|l|}{ Alcohol consumption } \\
\hline Low $^{a}$ & 263 & 1 [ref] & . & . & . & . \\
\hline Medium ${ }^{b}$ & 36 & 0.18 & $0.05-0.64$ & . & & . \\
\hline $\operatorname{High}^{c}$ & 25 & 0.80 & $0.37-1.71$ & . & & . \\
\hline Disease (self report diagnosis) & 340 & 1.83 & $1.25-2.67$ & . & & \\
\hline Past sickness absence & 334 & 1.01 & $1.01-1.02$ & . & & \\
\hline \multicolumn{7}{|l|}{ I Work environment } \\
\hline Job strain & 323 & 3.09 & $1.05-9.07$ & 293 & 1.16 & $0.38-3.53$ \\
\hline Psychological demands & 326 & 1.00 & $0.93-1.07$ & 294 & 0.97 & $0.90-1.05$ \\
\hline Decision latitude & 337 & 0.91 & $0.85-0.98$ & 303 & 0.97 & $0.90-1.05$ \\
\hline Social support at work & 318 & 0.93 & $0.85-1.02$ & 283 & 0.97 & $0.88-1.07$ \\
\hline Overcommitment & 329 & 0.99 & $0.95-1.04$ & 294 & 0.98 & $0.93-1.02$ \\
\hline |I Emotional support & 337 & 1.51 & $1.11-2.05$ & 301 & 1.74 & $1.21-2.51$ \\
\hline \multicolumn{7}{|l|}{ III Psychological resources } \\
\hline 1. Sense of coherence & 337 & 0.99 & $0.97-1.01$ & 302 & 1.01 & $0.99-1.04$ \\
\hline 2. Mastery & 330 & 1.00 & $0.94-1.06$ & 294 & 1.07 & $1.00-1.15$ \\
\hline 3. Self-esteem & 328 & 0.94 & $0.90-0.97$ & 293 & 0.98 & $0.94-1.02$ \\
\hline 4. Perceived control & 311 & 1.02 & $0.99-1.05$ & 280 & 1.05 & $1.02-1.09$ \\
\hline Previous back pain & 329 & 1.74 & $1.11-2.72$ & 295 & 1.43 & $0.92-2.22$ \\
\hline
\end{tabular}

The rate ratio in italics denotes significance $(p<0.05)$. (Result from Model II, see text). In model III rate ratios for confounders are not reported as separate regressions were run for each of the 10 psychosocial variables and previous back pain.

$\mathrm{n}=$ number of observations used; $\mathrm{RR}=$ rate ratio; $\mathrm{Cl}=$ confidence interval

a Low ( $<80 \mathrm{~g} /$ week for women, $<110 \mathrm{~g} /$ week for men)

${ }^{\mathrm{b}}$ Medium (between 80-139 $\mathrm{g} /$ week for women, 110-169 $\mathrm{g} /$ week for men)

${ }^{\mathrm{C}}$ High ( $\geq 140 \mathrm{~g} /$ week for women, $\geq 170 \mathrm{~g} /$ week for men)

${ }^{\dagger}$ Adjusted for age, sex; ${ }^{\ddagger}$ Adjusted for age, sex, BMl, smoking, alcohol consumption, SES, disease (self report of diagnosis), and past sickness absence.

defined activities [59], has been highlighted in the literature as playing an important role for work ability and in the process of returning to work $[60,61]$.

Just as could be seen for measures of sickness absence, high emotional support was related to poor prognosis of work ability in the multivariate logistic regression in Table 5. In this analysis, work ability is adjusted for age, sex, lifestyle factors, SES, disease, past sickness absence, previous back pain, work environment and psychological resources. In the analysis presented in Table 6, which was only

Table 5 Multivariate analysis of sickness absence spells and days and poor self assessed work ability

\begin{tabular}{lccc}
\hline Variables & Sick-leave spells $^{\mathbf{b}}$ & Sick-leave days $^{\mathbf{b}}$ & Poor work ability $^{\mathbf{c}}$ \\
\hline & $\mathbf{R R}^{\mathbf{a}} \mathbf{( 9 5 \% \mathbf { C l } )}$ & $\mathbf{R R}^{\mathbf{a}} \mathbf{( 9 5 \% \mathbf { C l } )}$ & $\mathbf{O R}^{\mathbf{a}}\left(\mathbf{9 5} \% \mathbf{C l}^{\mathbf{9}}\right)$ \\
\hline I Work environment & $1.09(0.95-1.25)$ & $1.00(0.84-1.19)$ & $1.39(0.98-1.98)$ \\
II Emotional support & $1.36(1.11-1.67)^{* *}$ & $1.68(1.22-2.31)^{* *}$ & $1.58(1.02-2.46)^{*}$ \\
III Psychological resources & $0.96(0.82-1.11)$ & $1.15(0.94-1.41)$ & $0.62(0.44-0.89)^{* *}$ \\
Previous back pain & $0.82(0.60-1.11)$ & $1.38(0.89-2.13)$ & $2.56(1.13-5.81)^{*}$ \\
\hline
\end{tabular}

$\mathrm{RR}=$ rate ratio; $\mathrm{OR}=$ odds ratio; $\mathrm{Cl}=$ confidence interval; ${ }^{*} p<0.05 ;{ }^{* *} p<0.01$;

${ }^{a}$ RRs and ORs are adjusted for age, sex, BMI, smoking, alcohol consumption, SES, disease (self report of diagnosis), past sickness absence, and all other variables in the table.

boisson regression.

'Logistic regression. 
Table 6 Prospective impact of psychosocial characteristics and previous back pain at baseline on self assessed "poor" work ability

\begin{tabular}{|c|c|c|c|c|c|c|}
\hline \multirow[t]{2}{*}{ Characteristic } & \multicolumn{3}{|c|}{$\begin{array}{l}\text { Age and sex adjusted } \\
(\text { Model II) }\end{array}$} & \multicolumn{3}{|c|}{$\begin{array}{l}\text { Adjusted for the final model } \\
\qquad(\text { Model III) }\end{array}$} \\
\hline & $\mathrm{n}$ & OR & $95 \% \mathrm{Cl}$ & $\mathbf{n}$ & OR & $95 \% \mathrm{Cl}$ \\
\hline \multicolumn{7}{|l|}{ Occupation (SES) } \\
\hline Non-manual & 185 & 1 [ref] & . & & . & . \\
\hline Manual & 112 & 2.56 & $1.35-4.87$ & & . & . \\
\hline Self-employed & 40 & 1.27 & $0.44-3.68$ & . & & \\
\hline Smoking, current & 332 & 1.46 & $0.73-2.90$ & & . & . \\
\hline BMI & 340 & 1.11 & $1.04-1.20$ & & . & . \\
\hline \multicolumn{7}{|l|}{ Alcohol consumption } \\
\hline Low $^{a}$ & 264 & 1 [ref] & . & . & & \\
\hline Medium ${ }^{b}$ & 36 & 0.91 & $0.33-2.55$ & & . & . \\
\hline High $^{c}$ & 25 & 2.33 & $0.84-6.51$ & . & . & \\
\hline Disease (self rep.diagnosis) & 341 & 1.09 & $0.61-1.96$ & & . & . \\
\hline Past sickness absence & 340 & 2.37 & $1.28-4.40$ & & . & . \\
\hline \multicolumn{7}{|l|}{ I Work environment } \\
\hline Job strain & 324 & 14.85 & $2.54-86.69$ & 298 & 11.71 & $1.69-81.01$ \\
\hline Psychological demands & 327 & 1.07 & $0.95-1.21$ & 299 & 1.07 & $0.93-1.22$ \\
\hline Decision latitude & 338 & 0.84 & $0.75-0.94$ & 309 & 0.83 & $0.73-0.95$ \\
\hline Social support at work & 319 & 0.88 & $0.76-1.02$ & 289 & 0.88 & $0.75-1.04$ \\
\hline Overcommitment & 330 & 1.00 & $0.94-1.07$ & 300 & 1.05 & $0.97-1.14$ \\
\hline || Emotional support & 338 & 1.28 & $0.90-1.81$ & 307 & 1.28 & $0.89-1.86$ \\
\hline \multicolumn{7}{|l|}{ III Psychological resources } \\
\hline 1. Sense of coherence & 338 & 0.96 & $0.93-0.99$ & 308 & 0.97 & $0.93-1.00$ \\
\hline 2. Mastery & 331 & 0.86 & $0.78-0.95$ & 300 & 0.84 & $0.76-0.94$ \\
\hline 3. Self-esteem & 329 & 0.90 & $0.85-0.96$ & 299 & 0.91 & $0.85-0.98$ \\
\hline 4. Perceived control & 312 & 0.95 & $0.91-0.99$ & 285 & 0.97 & $0.92-1.01$ \\
\hline Previous back pain & 330 & 2.78 & $1.31-5.92$ & 301 & 2.62 & $1.18-5.84$ \\
\hline
\end{tabular}

The odds ratio in italics denotes significance $(p<0.05)$. (Result from Model II, see text). In model III odds ratios for confounders are not reported as separate regressions were run for each of the 10 psychosocial variables and previous back pain.

$\mathrm{n}=$ number of observations used; $\mathrm{OR}=$ odds ratio; $\mathrm{Cl}=$ confidence interval.

a Low ( $<80 \mathrm{~g} /$ week for women, $<110 \mathrm{~g} /$ week for men)

${ }^{\mathrm{b}}$ Medium (between 80-139 $\mathrm{g} /$ week for women, 110-169 $\mathrm{g} /$ week for men)

${ }^{\mathrm{C}}$ High ( $\geq 140 \mathrm{~g} /$ week for women, $\geq 170 \mathrm{~g} /$ week for men)

${ }^{\dagger}$ Adjusted for age, sex; ${ }^{\ddagger}$ Adjusted for age, sex, BMl, smoking, alcohol consumption, SES, disease (self report of diagnosis), and past sickness absence.

adjusted for age, sex, lifestyle factors, SES, disease and past sickness absence, emotional support was not related to work ability. The results presented in Table 5 suggest that availability of emotional support provided by a person close's community outside work (family, friends, acquaintances), increases self-appraisal and boosts self-esteem, encourages to be absent from work.

A further question is how this applies to the perception of future work ability. It is possible to expect that absence in response to e.g. perceived strain at work, would actually reduce the risk of future inability to work. It is also possible that there is an element of reverse causality: that workers with a perception of decreased work ability may elicit more emotional support. This should be investigated further.

\section{Methodological considerations}

Several studies have shown that self-reported sickness absence is highly correlated with administrative information on such absence and have concluded that selfreported data are sufficiently valid measures for its correct assessment $[62,63]$. Furthermore, self-reported data provide information on the entire period of sickness absence, including also the first week of sickness absence, when no sickness absence certificate is needed.

In the analysis of psychosocial resources and previous back pain, all multivariate analyses were adjusted for SES (socioeconomic status measured as occupation), as SES might cause both workplace exposures and poor health [64]. The model that includes SES might be overadjusted because of the strong relation between 
occupation and psychosocial factors in the workplace $[10,15]$. The true relation between job strain, previous back pain, and sickness absence is probably between the unadjusted (Model I), and adjusted rate ratios (Model III).

The strength of the LSH study is its longitudinal design and a randomised sampling strategy. Several known and potential determinants, such as smoking, alcohol consumption, high BMI, and health status at baseline $[10,54,65,66]$, were controlled in the analysis, which therefore limited confounding bias. Health behaviour (i.e. smoking) may be part of the causal pathway linking exposures to psychosocial factors at work and sickness absence and adjustment for these factors might reduce the true effect of the psychosocial work environment on sickness absence. The analysis was adjusted for past sickness absence, as previous research has shown that sickness absence is a strong predictor of future absence [29].

A limitation was the relatively small size of the study population and the subsequent low statistical power, leading to a possible non identification of true effects. Another limitation resulted from loss of a number of participants because of non-response and because some individuals were no longer gainfully employed at follow-up. There was a pattern of non-response correlated with occupation that tended towards a healthy worker effect selection, again leading to a possible underestimation of true effects. The baseline work ability data is not available and thus it was not possible to study the association between work ability at baseline and work ability at follow-up. The potential limits of self reported measures in terms of common method variance or shared response biases may lead to an overestimation of associations between exposure and outcome variables. Negative affect could be mediating the effect of personality on absenteeism [67] and could affect the response, but this was not controlled for in the model. A final limitation is that these data do not provide information about how the exposure variables (work environment, emotional support, psychological resources, back/ neck pain) developed between baseline and follow-up

\section{Conclusions}

In a general middle aged working population high emotional support was related to more sickness absence and also poorer self assessed prognosis of work ability. Our findings suggest that both sickness absence and work ability are dependent of life outside work and can be affected by a person's close community (relatives, acquaintances, and friends).

\section{Acknowledgements}

The authors would like to express their appreciation to Dr Peter Garvin and Dr Johanna Lundberg, Linköping University, for their comments on the text.
The study was supported by a grant from the Department of Medical and Health Sciences, Linköping University, Sweden.

\section{Author details}

${ }^{1}$ Department of Medical and Health Sciences, Division of Community Medicine, Social Medicine and Public Health Science, Linköping University, Linköping, Sweden. ${ }^{2}$ Department of Medical and Health Sciences, Division of Physiotherapy, Linköping University, Linköping, Sweden.

\section{Authors' contributions}

NK, ES, and MK have made substantial contributions to the conception and design of this study and have contributed in the analysis and interpretation of data. NK has compiled data, produced tables, and has contributed to the statistical analysis. All authors have been involved in drafting the manuscript or revising it critically, and have read and approved its final version agreeing that it should be submitted for publication.

\section{Competing interests}

The authors declare that they have no competing interests.

Received: 9 June 2010 Accepted: 26 October 2010

Published: 26 October 2010

\section{References}

1. Vingård E: Health in Sweden: the National Public Health Report 2005. Chapter 5.6: Major public health problems-musculoskeletal disorders. Scand J Public Health Supp/ 2006, 67:104-112.

2. Karlsson NE, Carstensen JM, Gjesdal S, Alexanderson KA: Risk factors for disability pension in a population-based cohort of men and women on long-term sick leave in Sweden. Eur J Public Health 2008, 18:224-231.

3. Theorell T: I spåren av 90-talet [History, 20th century]. Stockholm: Karolinska Institutet University Press; 2006, (in Swedish)

4. Belkic KL, Landsbergis PA, Schnall PL, Baker D: Is job strain a major source of cardiovascular disease risk? Scand J Work Environ Health 2004, 30:85-128.

5. Stansfeld S, Candy B: Psychosocial work environment and mental healtha meta-analytic review. Scand J Work Environ Health 2006, 32:443-462.

6. Netterstrøm B, Conrad N, Bech P, Fink P, Olsen O, Rugulies R, Stansfeld S: The relation between work-related psychosocial factors and the development of depression. Epidemiol Rev 2008, 30:118-132.

7. Bonde JP: Psychosocial factors at work and risk of depression: a systematic review of the epidemiological evidence. Occup Environ Med 2008, 65:438-445.

8. Kivimäki M, Vahtera J, Ferrie JE, Hemingway H, Pentti J: Organisational downsizing and musculoskeletal problems in employees: a prospective study. Occup Environ Med 2001, 58:811-817.

9. Waddell G, Burton AK: Occupational health guidelines for the management of low back pain at work: evidence review. Occup Med 2001, 51:124-135.

10. North F, Syme SL, Feeney A, Head J, Shipley MJ, Marmot MG: Explaining socioeconomic differences in sickness absence: the Whitehall II study. BMJ 1993, 306:361-366.

11. North FM, Syme SL, Feeney A, Shipley M, Marmot M: Psychosocial work environment and sickness absence among British civil servants: the Whitehall II study. Am J Public Health 1996, 86:332-340.

12. Kivimäki $M$, Vahtera J, Thompson L, Griffiths A, Cox T, Pentti J: Psychosocial factors predicting employee sickness absence during economic decline. J Appl Psychol 1997, 82:858-872.

13. Kivimäki M, Elovainio M, Vahtera J, Ferrie JE: Organisational justice and health of employees: prospective cohort study. Occup Environ Med 2003, 60:27-34.

14. Niedhammer I, Bugel I, Goldberg M, Leclerc A, Guéguen A: Psychosocial factors at work and sickness absence in the Gazel cohort: a prospective study. Occup Environ Med 1998, 55:735-741.

15. Melchior M, Niedhammer I, Berkman LF, Goldberg M: Do psychosocial work factors and social relations exert independent effects on sickness absence? A six-year prospective study of the GAZEL cohort. J Epidemio/ Community Health 2003, 57:285-293.

16. Nielsen ML, Rugulies R, Christensen KB, Smith-Hansen L, Bjorner JB, Kristensen TS: Impact of the psychosocial work environment on registered absence from work: a two-year longitudinal study using the IPAW cohort. Work \& Stress 2004, 18:323-335. 
17. Karasek R, Theorell T: Healthy work: stress, productivity and the reconstruction of working life New York: Basic Books; 1990.

18. Andrea H, Beurskens AJ, Metsemakers JF, van Amelsvoort LG, van den Brandt PA, van Schayck CP: Health problems and psychosocial work environment as predictors of long term sickness absence in employees who visited the occupational physician and/or general practitioner in relation to work: a prospective study. Occup Environ Med 2003, 60:295-300.

19. Allebeck P, Mastekaasa A: Swedish Council on Technology Assessment in Health Care (SBU). Chapter 5. Risk factors for sick leave-general studies. Scand J Public Health Suppl 2004, 63:49-108.

20. Rugulies R, Christensen KB, Borritz M, Villadsen E, Bültmann U, Kristensen TS: The contribution of the psychosocial work environment to sickness absence in human service workers: Results of a three-year follow-up study. Work \& Stress 2007, 21:293-311

21. van Rhenen W, Schaufeli WB, van Dijk FJ, Blonk RW: Coping and sickness absence. Int Arch Occup Environ Health 2008, 81:461-472.

22. Rael EG, Stansfeld SA, Shipley M, Head J, Feeney A, Marmot M: Sickness absence in the Whitehall II study, London: the role of social support and material problems. J Epidemiol Community Health 1995, 49:474-481.

23. Kristensen TS: Sickness absence and work strain among Danish slaughterhouse workers: an analysis of absence from work regarded as coping behaviour. Soc Sci Med 1991, 32:15-27.

24. Lindberg $P$, Josephson $M$, Alfredsson $L$, Vingård E: Promoting excellent work ability and preventing poor work ability: the same determinants? Results from the Swedish HAKuL study. Occup Environ Med 2006, 63:113-120.

25. Hollman G, Kristenson M: The prevalence of the metabolic syndrome and its risk factors in a middle-aged Swedish population-mainly a function of overweight? Eur J Cardiovasc Nurs 2008, 7:21-26.

26. Lundberg J, Kristenson M: Is subjective status influenced by psychosocial factors? Soc Indic Res 2008, 89:375-390.

27. Lundberg J, Karlsson N, Kristenson M: Does two-year stability for scale scores of psychosocial factors differ by socioeconomic position? Psychol Rep 2009, 105:1009-1022

28. Garvin P, Nilsson L, Carstensen J, Jonasson L, Kristenson M: Circulating matrix metalloproteinase-9 is associated with cardiovascular risk factors in a middle-aged normal population. PLOS ONE 2008, 3:e1774.

29. Smulders PGW, Nijhuis FJN: The job demands-job control model and absence behaviour: results of a three-year longitudinal study. Work \& Stress 1999, 13:115-131.

30. Marhold C, Linton SJ, Melin L: Identification of obstacles for chronic pain patients to return to work: evaluation of a questionnaire. J Occup Rehabil 2002, 12:65-75.

31. Karasek RA: Job demands, job decision latitude, and mental strain: implications for job redesign. Adm Sci Q 1979, 24:285-308

32. Joksimovic L, Starke D, v d Knesebeck O, Siegrist J: Perceived work stress, overcommitment, and self-reported musculoskeletal pain: a crosssectional investigation. Int J Behav Med 2002, 9:122-138.

33. Undén $A-L$, Orth-Gomér $K$ : Development of a social support instrument for use in population surveys. Soc Sci Med 1989, 29:1387-1392.

34. Stansfeld S: Social support and social cohesion. In Social determinants of health. Edited by: Marmot M, Wilkinson RG. Oxford: Oxford University Press; 2006:147-171.

35. Antonovsky A: Unraveling the Mystery of Health: how people manage stress and stay well San Francisco: Jossey-Bass, CA; 1987.

36. Pearlin LI, Schooler C: The structure of coping. J Health Soc Behav 1978 19:2-21.

37. Skinner EA: A guide to constructs of control. J Pers Soc Psychol 1996, 71:549-570.

38. Skargren El, Öberg BE, Carlsson PG, Gade M: Cost and effectiveness analysis of chiropractic and physiotherapy treatment for low back and neck pain: Six-month follow-up. Spine 1997, 22:2167-2177.

39. Socio-economic classification (SEI). Vol 4. Reprinted 1984. Örebro: Statistiska centralbyrån (SCB)(Statistics Sweden). 1982, (In Swedish).

40. Christensen KB, Andersen PK, Smith-Hansen L, Nielsen ML, Kristensen TS: Analyzing sickness absence with statistical models for survival data. Scand J Work Environ Health 2007, 33:233-239.

41. Hair JF: Multivariate data analysis. 6 edition. Upper Saddle River, N.J. Pearson Prentice Hall; 2006.
42. Hochberg Y, Tamhane AC: Multiple comparison procedures New York: Wiley; 1987.

43. Best NG, Spiegelhalter DJ, Thomas A, Brayne CEG: Bayesian analysis of realistically complex models. J R Statist Soc A 1996, 159:323-342.

44. Brown GW, Harris T: Social origins of depression London: Tavistock Publications; 1978

45. House JS, Landis KR, Umberson D: Social relationships and health. Science 1988, 241:540-545.

46. ljzelenberg W, Burdorf A: Risk factors for musculoskeletal symptoms and ensuing health care use and sick leave. Spine 2005, 30:1550-1556.

47. Hansson T, Jensen I: Swedish Council on Technology Assessment in Health Care (SBU). Chapter 6. Sickness absence due to back and neck disorders. Scand J Public Health Suppl 2004, 63:109-151.

48. Bongers PM, de Winter CR, Kompier MA, Hildebrandt VH: Psychosocial factors at work and musculoskeletal disease. Scand J Work Environ Health 1993, 19:297-312.

49. Reiso H, Nygård JF, Brage S, Guldbrandson P, Tellness G: Work ability and duration of certified sickness absence. Scand J Public Health 2001, 29:218-225.

50. Alavinia SM, de Boer AG, van Duivenbooden JC, Frings-Dresen MH, Burdorf A: Determinants of work ability and its predictive value for disability. Occup Med 2009, 59:32-37.

51. Sell E, Bültman U, Rugulies R, Villadsen E, Faber A, Søgaard K: Predicting long-term sickness absence and early retirement pension from selfreported work ability. Int Arch Occup Environ Health 2009, 82:1133-1138.

52. Ilmarinen J: Work ability-a comprehensive concept for occupational health research and prevention [editorial]. Scand J Work Environ Health 2009, 35:1-5

53. IImarinen JE: Aging workers. Occup Environ Med 2001, 58:546-552.

54. van den Berg T, Alavinia S, Bredt F, Lindeboom D, Elders L, Burdorf A: The influence of psychosocial factors at work and life style on health and work ability among professional workers. Int Arch Occup Environ Health 2008, 81:1029-1036.

55. Bethge $M$, Radoschewski FM, Müller-Fahrnow W: Work stress and work ability: cross-sectional findings from the German sociomedical panel of employees. Disabil Rehab 2009, 31:1692-1699.

56. Martimo K-P, Varonen H, Husman K, Viikari-Juntura E: Factors associated with self-assessed work ability. Occup Med 2007, 57:380-382

57. Lillefjell M, Krokstad S, Espnes GA: Factors predicting work ability following multidisciplinary rehabilitation for chronic muskuloskeletal pain. J Occup Rehabil 2006, 16:543-555.

58. Kristenson M: Socio-economic position and health: the role of coping. In Social inequalities in health: new evidence and policy implications. Edited by: Siegrist J, Marmot M. Oxford University Press; 2006:127-151.

59. Bandura A: Self-efficacy: toward a unifying theory of behavioral change. Psychol Rev 1977, 84:191-215.

60. Labriola M, Lund T, Christensen KB, Albertsen K, Bültmann U, Jensen JN, Villadsen E: Does self-efficacy predict return-to-work after sickness absence? A prospective study among 930 employees with sickness absence for three weeks or more. Work 2007, 29:233-238.

61. Franche R-L, Krause N: Readiness for return to work following injury or illness: conceptualizing the interpersonal impact of health care, workplace, and insurance factors. J Occup Rehabil 2002, 12:233-256.

62. Ferrie JE, Kivimäki M, Head J, Shipley MJ, Vahtera J, Marmot MG: A comparison of self-reported sickness absence with absences recorded in employers' registers: evidence from the Whitehall II study. Occup Environ Med 2005, 62:74-79.

63. Voss M, Stark S, Alfredsson L, Vingård E, Josephson M: Comparisons of selfreported and register data on sickness absence among public employees in Sweden. Occup Environ Med 2008, 65:61-67.

64. Macleod J, Davey Smith G: Psychosocial factors and public health: a suitable case for treatment? J Epidemiol Community Health 2003 57:565-570.

65. Vahtera J, Poikolainen K, Kivimäki M, Ala-Mursula L, Pentti J: Alcohol intake and sickness absence: a curvilinear relation. Am J Epidemiol 2002, 156:969-976

66. Ferrie JE, Head J, Shipley MJ, Vahtera J, Marmot MG, Kivimäki M: BMI, obesity, and sickness absence in the Whitehall study. Obesity (Silver Spring) 2007, 15:1554-1564. 
67. Martocchio JJ, Jimeno Dl: Employee absenteeism as an affective event. Human Resourc Manage R 2003, 13:227-241.

\section{Pre-publication history}

The pre-publication history for this paper can be accessed here:

http://www.biomedcentral.com/1471-2458/10/648/prepub

doi:10.1186/1471-2458-10-648

Cite this article as: Karlsson et al:: Emotional support predicts more sickness absence and poorer self assessed work ability: a two-year prospective cohort study. BMC Public Health 2010 10:648.

Submit your next manuscript to BioMed Central and take full advantage of:

- Convenient online submission

- Thorough peer review

- No space constraints or color figure charges

- Immediate publication on acceptance

- Inclusion in PubMed, CAS, Scopus and Google Scholar

- Research which is freely available for redistribution

Submit your manuscript at www.biomedcentral.com/submit
C Biomed Central 